\title{
Biomarkers in Bone Metastases
}

\author{
Bülent KARABULUT
}

Department of Medical Oncology, Kent Health Group, Kent Oncology Center, Izmir-Turkey

\section{Introduction}

\section{Biochemical Markers of Bone Metabolism}

Approximately $70 \%$ of cancer patients, mainly those with breast and prostate cancer, tend to develop bone metastases during the disease course. Moreover, bone lesions could occur in patients with hematological malignancies, mainly multiple myeloma.[1-3] In addition to a large surface area, bones have several characteristics that allow the growth of cancer cells. The regulation of bone metabolism depends on the balance between the activity of osteoclasts and osteoblasts. Both osteolysis and osteogenesis are associated with the release of biochemical markers in the blood or urine.[4] Therefore, collagen peptides, the degradation products of osteolysis (e.g., amino [N]-and carboxy [C]-terminal cross-linked telopeptides of type I collagen or NTX and CTX), and biochemical markers of bone metabolism and terminal peptides cleaved from procollagen before integrating into the new bone matrix (e.g., procollagen type I N-terminal and C-terminal peptides [PINP and PICP]) could provide significant insights into the effects of tumor growth on bone turnover.[5] While serum levels and urinary concentration reflect the rate of osteolysis, the serum levels of bone-specific alkaline phosphatase (bone ALP) reflect the rate of osteogenesis. Furthermore, certain bone metabolism markers could be associated with both osteolysis and osteogenesis (e.g., osteocalcin).[5] Although changes in bone biomarkers are not disease-specific, these are associated with the changes in bone metabolism independent of the underlying cause.[4]

Osteoprotegerin (OPG) and osteopontin (e.g., it is one of the valuable biomarkers due to the effects of parathyroid hormone on bones) are possibly associated with prognosis in patients with bone metastases.

\section{Prognosis}

\section{Bone Marker Levels and Risk of Clinical Events}

Because the levels of bone markers reflect the bone turnover, they could provide important clinical information on patients' risks of skeletal-related events (SREs) and death. Consequently, assessing the bone markers could identify patients requiring intense follow-ups and/or interventions (currently, level 2 evidence). For example, a substantial increase in the levels of bone resorption markers could be an early indicator of an upcoming fracture in patients with metastatic castration-resistant prostate cancer (CRPC), which could provide a basis for the early application of preventive approaches.[6] Data on the levels of bone markers with clinical outcomes are summarized below.

\section{NTX and Bone ALP}

Phase III trials have been conducted on zoledronic acid to study its association with prognosis by monitoring the levels of bone markers in patients with multiple myeloma and breast cancer $(n=1,648)$, CRPC $(n=643)$, and lung cancer or other solid tumors $(n=773)$ and bone metastases. [7-9] Increases in the levels of bone markers at baseline and during the trial in patients with solid tumors and bone metastases were associated with SREs, disease progression, and increased risk of death.[5,7] Although statistical heterogeneity was not reported for outcomes, increases in the levels of bone marker compared to the baseline revealed a statistically more significant correlation. For example, when patients with baseline NTX levels equal to or above $100 \mathrm{nmol} / \mathrm{mmol}$ and those with lower NTX levels were compared, the RR of SREs was found to be 1.59 (95\% confidence interval $[\mathrm{CI}]=1.17,2.14 ; \mathrm{p}=0.003)$. Simi- 
larly, an increase in the ALP during the study has also been associated with an increased risk of disease progression and death.[10] In these phase III trials, similar correlations were recorded between the levels of bone markers and clinical outcomes in patients receiving bisphosphonate treatment. In these studies, increases in NTX and bone ALP levels in patients with different solid tumors and bone metastases were significantly correlated with the increased risks of SREs, death, and disease progression.[11] In these analyses, NTX levels were classified as high $(100 \mathrm{nmol} / \mathrm{mmol}$ creatinine $)$, medium (50-99 nmol/mmol creatinine), and low $(<50$ $\mathrm{nmol} / \mathrm{mmol}$ creatinine). The strongest correlation with clinical outcomes was demonstrated when patients with high NTX levels during the trial were compared with those with low levels. Although the correlation pattern between NTX levels and clinical outcomes was similar in all patients, the strongest correlation was found in those with breast and prostate cancer. In addition, the correlation between serum bone ALP levels (categorized as high and low using a cut-off value of $146 \mathrm{U} / \mathrm{L}$ correspondings to the upper limit of normal for that test) and the risks of SREs, disease progression, and death was assessed in these analyses. A strong correlation was found with high bone ALP levels in this trial.[11] Furthermore, a significant correlation was found between high levels of bone marker and the risks of skeletal complications and death during data analyses of CRPC patients included in the ZOL phase III trial.[12,13] The risk of death increased by more than 2.5-fold with the NTX levels equal to or higher than $180.5 \mathrm{nmol} / \mathrm{mmol}(\mathrm{RR}=2.58 ; 95 \% \mathrm{CI}=1.92,3.47$; $\mathrm{p}<0.001)$. The results were obtained by comparing the data of patients with high and low $(<54.5 \mathrm{nmol} / \mathrm{mmol})$ baseline NTX levels.[13]

When assessed as a continuous variable, every increase of $50 \mathrm{nmol} / \mathrm{mmol}$ compared to the baseline NTX levels was associated with a $5 \%$ increase in the risk of death $(\mathrm{p}<0.001)$. In addition, each increase of $200 \mathrm{U} / \mathrm{L}$ compared to the baseline bone ALP levels was associated with a $4 \%$ increase in the risk of death $(\mathrm{p}<0.001)$.

\section{Other Markers}

In addition to NTX and bone ALP, certain other markers could have prognostic value in patients with bone metastases. However, there is limited evidence for various markers, including OPG, PINP, and TRAcP-5B. [14-19] Recent evidence suggests that PINP is particularly a promising marker for metastatic bone disease. For example, baseline PINP levels were approxi- mately 2.3-fold higher in patients with bone metastases compared to those without bone metastases and with breast or ovarian cancer $(n=100)$.[20] In a study, oral clodronate or placebo was administered as an adjuvant for two years in addition to the standard therapy in patients diagnosed with breast cancer. In the retrospective subgroup analyses of this study, including 851 female patients, increases in PINP levels within the first year of treatment were possibly associated with the long-term risk of development of bone metastasis. [21] In a study in patients with PC ( $n=65)$, PINP levels were more strongly correlated than the PSA levels with the development of bone metastases.[16] Nevertheless, to date, the majority of the studies have not assessed the levels of these markers. Therefore, the usefulness of these markers can be confirmed by analyzing the data obtained from large patient populations.

\section{Importance of Normalization of Bone Markers Dur- ing Treatment}

Data obtained from the ZOL phase III trials showed a substantial decrease in the risk of death in patients who experienced a decrease from high baseline NTX levels to normal NTX levels within three months compared to those who had continuously increasing NTX levels (a decrease of $48 \%$ to $59 \%$ depending on the type of the tumor; all of the population [ $<<0.02])$.[22] In addition, similar correlations were shown between the normalization of NTX levels and SRE-free or bone lesion progression-free survival data. Similarly, in the subgroup analyses in patients with breast cancer, normalization of NTX levels within 3 months was associated with a decrease of approximately $50 \%$ in the risk of first SRE $(\mathrm{RR}=0.504 ; 95 \% \mathrm{CI}=0.318,0.798 ; \mathrm{p}=0.0034)$, and the risk of first pathological fracture or orthopedic surgery $(\mathrm{RR}=0.486 ; 95 \% \mathrm{CI}=0.294,0.805 ; \mathrm{p}=0.005)$ was significantly low. [23] Furthermore, a decrease in the NTX levels has been reported to decrease the risk of a negative clinical outcome.

Changes in bone markers were examined in randomized phase II trials of denosumab in patients with bone metastases due to prostate, breast, or other solid tumors. Among female patients with a diagnosis of breast cancer and bone metastasis $(n=255)$, more than $65 \%$ of the decrease in the NTX levels was detected up to the 13 th week in $74 \%$ of the patients who were on denosumab.[24] In the second trial, denosumab treatment was associated with fewer total SREs. However, none of these trials examined the correlation between 
the risk of SRE and the decrease in NTX levels. Therefore, data obtained from phase III trials with longer follow-ups are required.

Several studies have demonstrated that the levels of bone markers could provide prognostic information in patients with malignant bone diseases. Although the greatest evidence to date is for NTX and bone ALP, certain studies have reported that PINP could be useful as well. Nevertheless, several questions remain to be answered, including those about identifying the "normal" and "high" levels of markers, identifying the most appropriate marker among the markers measured in serum or urine, optimal timing of bone marker assessments, and lack of consensus on the optimal intervals of bone marker kinetic measurements.[25] Adjuvant therapies might considerably change the levels of bone markers in early-stage cancer patients, especially in patients with breast or prostate cancer. Most aromatase inhibitor therapies are associated with a substantial increase in bone resorption several months after the treatment.[26] There is evidence showing that early increases in the NTX and bone ALP levels predicted long-term bone loss in postmenopausal early-stage breast cancer. However, sufficient data are not available to support their use as indicators of fractures. [27] Androgen deprivation therapy (ADT) using gonadotropin-releasing hormone agonists in patients with hormone-resistant prostate cancer is associated with simultaneous increases in bone loss and levels of bone markers. [28,29] Androgen deprivation increases the levels of NTX and bone ALP in hormone-naive patients as well as in patients with non-metastatic prostate cancer.[30] In addition, bone ALP levels greatly increased in patients who developed bone metastasis and received $\mathrm{ADT}$ compared to those without skeletal involvement who received ADT.[30] In contrast, anti-androgen therapy (e.g., bicalutamide monotherapy) did not increase the levels of bone markers.[30] Furthermore, bone marker levels are affected by antiresorptive therapies used to prevent bone loss during ADT.[3133] Nevertheless, these are preliminary studies, and more data are required to confirm the correlations between bone marker levels and the risk of bone loss and fractures during hormone therapy for cancer. Furthermore, the potential use of elevated levels of bone markers to predict the development of bone metastases is yet to be studied in an adjuvant setting, and more studies are warranted to show the importance of bone marker assessments in patients without bone metastases.

\section{Pre-treatment Bone Marker Levels Identify Which Patients Will Benefit from Bone-targeted Therapy}

Bone marker assessments might provide valuable information on metastatic bone diseases and aggressiveness. Similarly, several analyses have revealed the prognostic importance of bone markers in patients receiving bone-targeted therapy. Although the available data correlating the bone marker levels with treatment benefits are limited, early studies with NTX and bone ALP in patients with other solid tumors (excluding breast and prostate cancers) provided the rationale to examine these types of correlations further.[19,34,35] For example, the analgesic effect of pamidronate was reduced in patients with painful bone metastases showing normalization in NTX or CTX levels compared to those who did not show normalization in these levels. [36] The latest reports demonstrated that patients with high NTX levels that normalized during zoledronic acid treatment had better survival compared to those with high NTX levels.[22] Nonetheless, patients with high baseline NTX levels (an indicator of increased disease burden in bones) could benefit more from bonetargeted therapies as they are at higher risk for SREs, which potentially restrict daily life. Additional analyses were performed using the data obtained from patients enrolled in these studies to further examine the correlations between bone marker levels and clinical outcomes. A multivariable analysis in patients with bone metastasis due to non-small cell lung cancer (NSCLC) provided level 2 evidence that baseline NTX levels furnished information on the clinical benefits obtained with zoledronic acid treatment. Patients with bone metastasis due to NSCLC enrolled in phase III, multi-center, randomized, placebo-controlled zoledronic acid trial ( $4 \mathrm{mg}$ by a 15-minute infusion every three weeks up to 21 months) were included in this analysis, and analyses were performed using baseline NTX levels. [9,37] In this first study on the use of zoledronic acid in patients with NSCLC, the risk of SRE decreased substantially ( $\mathrm{p}=0.028$ ).[38] In the sub-group analysis of 262 patients with baseline NTX assessment, the risk of death was similar in patients with normal baseline NTX levels $(<64 \mathrm{nmol} / \mathrm{mmol}$ creatinine; $\mathrm{n}=118)$ whether they received zoledronic acid or placebo $(\mathrm{RR}=1.326 ; \mathrm{p}=0.223)$. In contrast, zoledronic acid reduced the risk of death by $35 \%$ compared to placebo $(\mathrm{RR}=0.652 ; \mathrm{p}=0.025)$ in patients with high baseline NTX levels $(64 \mathrm{nmol} / \mathrm{mmol}$ creatinine; $\mathrm{n}=144)$.[38] A meta-analysis of data obtained from three phase III trials on zoledronic acid in patients with BC, CRPC, or 
lung cancer and other solid tumors was performed to examine further the effects of zoledronic acid on the survival of patients with metastatic bone disease and increased bone resorption $(n=1,642)$.[38] These analyses only included patients with available baseline bone marker assessments and baseline data for all common variables. The results showed that the predictive benefits of bone markers in patients with metastatic bone disease provided level 3 evidence. In patients with high NTX levels (64 nmol/mmol creatinine), zoledronic acid substantially reduced the risk of death by $17 \%$ compared to placebo $(R R=0.828 ; p=0.034)$.[38] Similar results were reported for patients with considerably high baseline NTX levels $(100 \mathrm{nmol} / \mathrm{mmol}$ creatinine), and zoledronic acid substantially reduced the risk of death by $26 \%$ compared to placebo $(\mathrm{RR}=0.736$; $\mathrm{p}=0.006$ ).[39] Nevertheless, the survival benefit was insignificant in patients with normal baseline NTX levels who received zoledronic acid treatment.

Although these results are encouraging, only retrospective analyses provide a correlation between the biochemical markers of bone turnover and clinical outcomes. These findings show that pre-treatment assessments of NTX and bone ALP levels in patients who received bisphosphonate for bone metastases could be useful in studying disease progression. Nevertheless, prospective studies are required to confirm the importance of NTX (or other bone markers) for survival benefits obtained from bone-targeted therapies.

\section{Bone Marker Levels and Treatment Decisions}

Pharmacokinetic studies demonstrated that bisphosphonates are rapidly cleared from blood circulation and absorbed by the skeletal system and are particularly concentrated in bone resorption areas.[39-41] Nevertheless, the concentration of bisphosphonates in the bone tissue of patients with metastatic bone disease considerably varies among patients. The long-lasting antiresorptive activity of bone-targeted agents shows that these agents are stored in the bone for a prolonged duration. Osteolysis is not only associated with the release of growth factors from the bone matrix but also releases the stored bisphosphonates. [42] Therefore, bone resorption rate during treatment depends on several factors, including bisphosphonate skeletal retention, matrix-associated bisphosphonate loss, and reuptake process-"recycling"-by osteoclasts.[43-45] Therefore, bone marker levels during the treatment could reflect an integrated index of the effect of bisphosphonates on osteoclast-mediated bone resorption. The continuous increase in the levels of bone marker could reflect the need for more intense treatment in patients with malignant bone diseases. Based on the evidence obtained from phase III registry studies, current clinical practice guidelines support the use of bisphosphonate treatment according to the dosing schedules approved for up to 2 years in patients with malignant bone diseases. Treatment models longer than two years are variable, with only anecdotal data showing that low-frequency bisphosphonate treatment is sufficient to protect skeletal health for longer than two years. Another possibility is discontinuing bone-targeted agents during the asymptomatic period and reinitiating the treatment in case symptoms reoccur. Patient-based strategies should minimize potential side effects and optimize treatment efficacy. OPTIMIZE-2 trial $(n=650)$ assessed the efficacy of zoledronic acid administered monthly or every three months for 48 weeks in 290 random patients with breast cancer and bone metastasis who had been previously treated with intravenous bisphosphonate (9 doses).[46] In this study, an important endpoint was the assessment of monthly changes in the levels of bone markers of both treatment arms (monthly or every three months ZOL). Nevertheless, more such trials are required in patients with different types of cancers to elucidate the potential of changes in the levels of bone markers to select the right treatment strategy.

\section{Detection of Cancer-related Bone Diseases: Do Bone Markers Have a Role in Screening Bone Lesions?}

Several small trials have reported the correlations between bone biomarker levels and bone involvement/ bone disease progression in various malignancies. Nonetheless, more research and trials are required before bone markers are integrated into routine screening protocols for malignant bone diseases.

\section{Information Obtained from Non-bone Markers}

Bone sialoprotein (BSP) is a tumor marker associated with skeletal metastasis. Preclinical data show that it mediates the invasiveness to stroma surrounding the breast and pancreatic cancer cells, thereby facilitating metastasis.[47,48] A small trial in patients with breast cancer $(n=39)$ reported higher levels of BSP in primary breast cancer to correlate with the risk of bone metastasis $(p=0.008)$.[49] In addition, in a larger trial 
that examined the paraffin blocks of 454 patients diagnosed with breast cancer, elevated levels of BSP in the tumor tissue had prognostic importance for shorter survival, especially in patients with lymph node-negative diseases.[50] Moreover, bone metastasis developed in $22 \%$ of patients with BSP-positive tumors.[50] In addition, another study reported that the expression of BSP in the primary tumor was strongly correlated with the development of bone metastasis $(\mathrm{p}<0.001)$, and the overall survival results were poor in these patients $(\mathrm{p}=0.02)$.[51] Furthermore, serum BSP levels correlated with metastasis-free survival ( $p=0.03)$. [52] Lastly, the ectopic expression of dentin sialophosphoprotein (which shares structural and genetic similarities with BSP) in the prostate cancer tissue correlated with the pathological stage $(\mathrm{p}=0.0087)$ and high Gleason score $(\mathrm{p}=0.0176)$.[53] In addition, the study on enzymes associated with the metastatic process can provide information on the disease course. For example, a pilot study in patients with bone metastasis due to breast cancer indicated that high levels of matrix metalloproteinase (MMP)-9 during the initiation of bisphosphonate treatment were associated with a shorter time to develop the symptomatic bone disease (i.e., SREs).[54]

PSA is a standard diagnostic and follow-up tool in prostate cancer. A trial including non-metastatic prostate cancer patients with increasing PSA despite ADT ( $\mathrm{n}=201$ ) with a baseline PSA level above $10 \mathrm{ng} /$ $\mathrm{mL}$ and a high PSA rate (PSA increase rate over time) reported a three-fold or more increase in the risk of bone metastasis ( $\mathrm{p}<0.001$ for each). The survival was found to be low in these patients.[55] In other trials, LDH levels, alpha-1-chymotrypsin-PSA complex, and HER-2/neu were identified as potential prognostic factors in prostate cancer with bone metastasis. [56-58] However, further trials with larger patient populations are required.

\section{Conclusions}

Several trials have evaluated the possible roles of bone turnover markers of bone diseases in the diagnosis, prognosis, and patient follow-ups. Considering the heterogeneity of available markers and their levels in different types of cancers, routine use of bone markers in clinical practice is not recommended at this stage. Moreover, bone markers or bone marker panels in combination with disease-specific markers (e.g., PSA) may prove to be more beneficial than a single bone marker. Nevertheless, bone markers can provide more useful information in addition to other established diagnostic and follow-up methods.

- Level 1 evidence: Bone marker assessments have an established role in the preclinical and early clinical development of bone-targeted therapies. Moreover, they provide appropriate, relatively non-invasive tools during dose-ranging and pharmacodynamic studies.

- Level 2 evidence: Bone marker levels may be beneficial to predict the progression of bone metastases, the ongoing risk of SREs, and mortality in patients with the malignant bone disease (in the presence or absence of bisphosphonate treatment). The most beneficial markers to date are NTX in multiple myeloma and breast cancer and bone ALP in CRPC.

- Level 2 evidence: Early changes in the bone marker levels during bisphosphonate treatment might predict long-term benefits.

- Level 3 evidence: Currently, bone markers are not adequately sensitive to safely detect the bone metastases individually, to compare the efficacies of different bone-targeted therapies, or to drive the dosage and frequency of bisphosphonate treatment in patients with malignant bone disease.

- Further prospective trials are required.

Assessing the correlations between the changes in the levels of bone markers and clinical outcomes in an individual and not population could be significant in

- comparing more than one marker in a single study,

- developing markers to estimate the future risk of bone metastases,

- adapting the bisphosphonate treatment for individual patients,

- evaluating the non-bisphosphonate bone-targeted agents and their effects on bone metabolism,

- standardizing the laboratory tests for bone markers, and

- precisely determining the "normal" and "high" levels of bone markers in patients with malignant bone lesions.

In brief, research in the field of bone markers is rapidly expanding. Although the absolute levels of bone markers have prognostic significance for patients with bone metastases or multiple myeloma and early reductions in the levels of bone markers during bisphosphonate treatment, have predicted the therapeutic effect, the role and benefit of bone markers in guiding therapeutic decisions for individual patients remain to be determined. Therefore, bone marker data could assist in assessing the risk of worsening skeletal health 
of a patient. However, bone markers are not ready to be used instead of established diagnostic and follow-up techniques yet.

\section{References}

1. Kriege M, Seynaeve C, Meijers-Heijboer H, Collee JM, Menke-Pluymers MB, Bartels CC, et al. Distant disease-free interval, site of first relapse and post-relapse survival in BRCA1-and BRCA2-associated compared to sporadic breast cancer patients. Breast Cancer Res Treat 2008;111(2):303-11.

2. Hess KR, Varadhachary GR, Taylor SH, Wei W, Raber MN, Lenzi R, et al. Metastatic patterns in adenocarcinoma. Cancer 2006;106(7):1624-33.

3. Uchida A, Wakabayashi H, Okuyama N, Okamura A, Matsumine A, Kusuzaki K. Metastatic bone disease: pathogenesis and new strategies for treatment. J Orthop Sci. 2004;9(4):415-20.

4. Fohr B, Dunstan CR, Seibel MJ. Clinical review 165: Markers of bone remodeling in metastatic bone disease. J Clin Endocrinol Metab. 2003;88(11):5059-75.

5. Seibel MJ. Clinical use of markers of bone turnover in metastatic bone disease. Nat Clin Pract Oncol 2005;2(10):504-17; quiz $1 \mathrm{p}$ following 533.

6. Smith MR, Cook RJ, Coleman R, Brown J, Lipton A, Major P, et al. Predictors of skeletal complications in men with hormone-refractory metastatic prostate cancer. Urology 2007;70(2):315-9.

7. Rosen LS, Gordon D, Kaminski M, Howell A, Belch A, Mackey J, Apffelstaedt J, et al. Long-term efficacy and safety of zoledronic acid compared with pamidronate disodium in the treatment of skeletal complications in patients with advanced multiple myeloma or breast carcinoma: a randomized, double-blind, multi-center, comparative trial. Cancer 2003;98(8):1735-44.

8. Saad F, Gleason DM, Murray R, Tchekmedyian S, Venner P, Lacombe L, et al. Long-term efficacy of zoledronic acid for the prevention of skeletal complications in patients with metastatic hormone-refractory prostate cancer. J Natl Cancer Inst 2004;96(11):87982.

9. Rosen LS, Gordon D, Tchekmedyian NS, Yanagihara R, Hirsh V, Krzakowski M, et al. Long-term efficacy and safety of zoledronic acid in the treatment of skeletal metastases in patients with non-small cell lung carcinoma and other solid tumors: a randomized, Phase III, double-blind, placebo-controlled trial. Cancer 2004;100(12):2613-21.

10. Brown JE, Cook RJ, Major P, Lipton A, Saad F, Smith $\mathrm{M}$, et al. Bone turnover markers as predictors of skele- tal complications in prostate cancer, lung cancer, and other solid tumors. J Natl Cancer Inst 2005;97(1):5969.

11. Coleman RE, Major P, Lipton A, Brown JE, Lee KA, Smith $\mathrm{M}$, et al. Predictive value of bone resorption and formation markers in cancer patients with bone metastases receiving the bisphosphonate zoledronic acid. J Clin Oncol 2005;23(22):4925-35.

12. Lorente JA, Valenzuela H, Morote J, Gelabert A. Serum bone alkaline phosphatase levels enhance the clinical utility of prostate specific antigen in the staging of newly diagnosed prostate cancer patients. Eur J Nucl Med 1999;26(6):625-32.

13. Cook RJ, Coleman R, Brown J, Lipton A, Major P, Hei YJ, et al. Markers of bone metabolism and survival in men with hormone-refractory metastatic prostate cancer. Clin Cancer Res 2006;12(11 Pt 1):3361-7.

14. Jung K, Lein M, Stephan C, Von Hösslin K, Semjonow A, Sinha P, et al. Comparison of 10 serum bone turnover markers in prostate carcinoma patients with bone metastatic spread: diagnostic and prognostic implications. Int J Cancer 2004;111(5):783-91.

15. Leeming DJ, Koizumi M, Byrjalsen I, Li B, Qvist P, Tankó LB. The relative use of eight collagenous and noncollagenous markers for diagnosis of skeletal metastases in breast, prostate, or lung cancer patients. Cancer Epidemiol Biomarkers Prev 2006;15(1):32-8.

16. Koizumi M, Yonese J, Fukui I, Ogata E. The serum level of the amino-terminal propeptide of type I procollagen is a sensitive marker for prostate cancer metastasis to bone. BJU Int 2001;87(4):348-51.

17. Koopmans N, de Jong IJ, Breeuwsma AJ, van der Veer E. Serum bone turnover markers (PINP and ICTP) for the early detection of bone metastases in patients with prostate cancer: a longitudinal approach. J Urol 2007;178(3 Pt 1):849-53; discussion 853; quiz 1129.

18. Chung YC, $\mathrm{Ku} \mathrm{CH}$, Chao TY, Yu JC, Chen MM, Lee $\mathrm{SH}$. Tartrate-resistant acid phosphatase $5 \mathrm{~b}$ activity is a useful bone marker for monitoring bone metastases in breast cancer patients after treatment. Cancer Epidemiol Biomarkers Prev 2006;15(3):424-8.

19. Koizumi M, Takahashi S, Ogata E. Comparison of serum bone resorption markers in the diagnosis of skeletal metastasis. Anticancer Res 2003;23(5b):40959.

20. Oremek G, Sauer-Eppel H, Klepzig M. Total procollagen type 1 amino-terminal propeptide (total P1NP) as a bone metastasis marker in gynecological carcinomas. Anticancer Res 2007;27(4A):1961-2.

21. McCloskey E, Paterson A, Kanis J, Tähtelä R, Powles T. Effect of oral clodronate on bone mass, bone turnover 
and subsequent metastases in women with primary breast cancer. Eur J Cancer 2010;46(3):558-65.

22. Lipton A, Cook R, Saad F, Major P, Garnero P, Terpos $\mathrm{E}$, et al. Normalization of bone markers is associated with improved survival in patients with bone metastases from solid tumors and elevated bone resorption receiving zoledronic acid. Cancer 2008;113(1):193201.

23. Lipton A, Cook RJ, Major P, Smith MR, Coleman RE. Zoledronic acid and survival in breast cancer patients with bone metastases and elevated markers of osteoclast activity. Oncologist 2007;12(9):1035-43.

24. Lipton A, Steger GG, Figueroa J, Alvarado C, SolalCeligny P, Body JJ, et al. Randomized active-controlled phase II study of denosumab efficacy and safety in patients with breast cancer-related bone metastases. J Clin Oncol 2007;25(28):4431-7.

25. Coleman R, Brown J, Terpos E, Lipton A, Smith MR, Cook $\mathrm{R}$, et al. Bone markers and their prognostic value in metastatic bone disease: clinical evidence and future directions. Cancer Treat Rev 2008;34(7):629-39.

26. Goss PE, Hadji P, Subar M, Abreu P, Thomsen T, Banke-Bochita J. Effects of steroidal and nonsteroidal aromatase inhibitors on markers of bone turnover in healthy postmenopausal women. Breast Cancer Res 2007;9(4):R52.

27. Brufsky A, Harker G, Beck T, Carroll R, Warsi G, Jin L, et al. Bone-specific alkaline phosphatase (BSAP) and serum N-telopeptide (sNTX) as predictors of bone loss in postmenopausal women with early breast cancer receiving letrozole as adjuvant therapy: a 5-year study (Z-FAST). Cancer Res 2009;69(Suppl.):176s [abstract 2067].

28. Greenspan SL, Coates P, Sereika SM, Nelson JB, Trump DL, Resnick NM. Bone loss after initiation of androgen deprivation therapy in patients with prostate cancer. J Clin Endocrinol Metab 2005;90(12):6410-7.

29. Smith MR, Fallon MA, Goode MJ. Cross-sectional study of bone turnover during bicalutamide monotherapy for prostate cancer. Urology 2003;61(1):127-31.

30. Michaelson MD, Marujo RM, Smith MR. Contribution of androgen deprivation therapy to elevated osteoclast activity in men with metastatic prostate cancer. Clin Cancer Res 2004;10(8):2705-8.

31. Ishizaka K, Machida T, Kobayashi S, Kanbe N, Kitahara S, Yoshida K. Preventive effect of risedronate on bone loss in men receiving androgen-deprivation therapy for prostate cancer. Int J Urol 2007;14(12):1071-5.

32. Israeli RS, Rosenberg SJ, Saltzstein DR, Gottesman JE, Goldstein HR, Hull GW, et al. The effect of zoledronic acid on bone mineral density in patients undergo- ing androgen deprivation therapy. Clin Genitourin Cancer 2007;5(4):271-7.

33. Magno C, Anastasi G, Morabito N, Gaudio A, Maisano D, Franchina F, et al. Preventing bone loss during androgen deprivation therapy for prostate cancer: early experience with neridronate. Eur Urol 2005;47(5):575-80; discussion 580-1.

34. Ali SM, Demers LM, Leitzel K, Harvey HA, Clemens $\mathrm{D}$, Mallinak N, et al. Baseline serum NTx levels are prognostic in metastatic breast cancer patients with bone-only metastasis. Ann Oncol 2004;15(3):455-9.

35. Hegele A, Wahl HG, Varga Z, Sevinc S, Koliva L, Schrader AJ, et al. Biochemical markers of bone turnover in patients with localized and metastasized prostate cancer. BJU Int 2007;99(2):330-4.

36. Vinholes JJ, Purohit OP, Abbey ME, Eastell R, Coleman RE. Relationships between biochemical and symptomatic response in a double-blind randomised trial of pamidronate for metastatic bone disease. Ann Oncol 1997;8(12):1243-50.

37. Hirsh V, Major PP, Lipton A, Cook RJ, Langer CJ, Smith MR, et al. Zoledronic acid and survival in patients with metastatic bone disease from lung cancer and elevated markers of osteoclast activity. J Thorac Oncol 2008;3(3):228-36.

38. Coleman R, Cook R, Saad F, Hirsh V, Major P, Dias $\mathrm{R}$, et al. Meta-analysis of effects of zoledronic acid on survival in patients with metastatic bone disease (MBD) and high bone turnover. Cancer Treat Rev 2008;34(Suppl 1):S86 [abstract P81].

39. Chen T, Berenson J, Vescio R, Swift R, Gilchick A, Goodin S, et al. Pharmacokinetics and pharmacodynamics of zoledronic acid in cancer patients with bone metastases. J Clin Pharmacol 2002;42(11):1228-36.

40. Sato M, Grasser W, Endo N, et al. Bisphosphonate action. Alendronate localization in rat bone and effects on osteoclast ultrastructure. J Clin Invest 1991;88(6):2095-105.

41. Azuma Y, Sato H, Oue Y, Okabe K, Ohta T, Tsuchimoto $\mathrm{M}$, et al. Alendronate distributed on bone surfaces inhibits osteoclastic bone resorption in vitro and in experimental hypercalcemia models. Bone 1995;16(2):235-45.

42. Chirgwin JM, Mohammad KS, Guise TA. Tumor-bone cellular interactions in skeletal metastases. J Musculoskelet Neuronal Interact 2004;4(3):308-18.

43. Cremers SC, Papapoulos SE, Gelderblom H, Seynaeve $C$, den Hartigh J, Vermeij P, et al. Skeletal retention of bisphosphonate (pamidronate) and its relation to the rate of bone resorption in patients with breast cancer and bone metastases. J Bone Miner Res 
2005;20(9):1543-7.

44. Nancollas GH, Tang R, Phipps RJ, Henneman Z, Gulde S, Wu W, et al. Novel insights into actions of bisphosphonates on bone: differences in interactions with hydroxyapatite. Bone 2006;38(5):617-27.

45. Russell RG. Determinants of structure-function relationships among bisphosphonates. Bone 2007;40(Suppl 2):S21-5.

46. U.S. National Institutes of Health. Efficacy and safety of zoledronic acid (every 4 weeks vs. every 12 weeks) in patients with documented metastases from bone cancer. http://www.clinicaltrials.gov/ct2/show/ NCT00320710 [accessed 18.08.10].

47. Nam JS, Suchar AM, Kang MJ, Stuelten CH, Tang B, Michalowska AM, et al. Bone sialoprotein mediates the tumor cell-targeted prometastatic activity of transforming growth factor beta in a mouse model of breast cancer. Cancer Res 2006;66(12):6327-35.

48. Kayed H, Kleeff J, Keleg S, Felix K, Giese T, Berger MR, et al. Effects of bone sialoprotein on pancreatic cancer cell growth, invasion and metastasis. Cancer Lett 2007;245(1-2):171-83.

49. Bellahcene A, Kroll M, Liebens F, Castronovo V. Bone sialoprotein expression in primary human breast cancer is associated with bone metastases development. J Bone Miner Res 1996;11(5):665-70.

50. Bellahcène A, Menard S, Bufalino R, Moreau L, Castronovo $\mathrm{V}$. Expression of bone sialoprotein in primary human breast cancer is associated with poor survival. Int J Cancer 1996;69(4):350-3.

51. Papotti M, Kalebic T, Volante M, Chiusa L, Bacillo $\mathrm{E}$, Cappia S, et al. Bone sialoprotein is predictive of bone metastases in resectable non-small-cell lung cancer: a retrospective case-control study. J Clin Oncol 2006;24(30):4818-24.

52. Loibl S, Königs A, Kaufmann M, Costa SD, Bischoff
J. Bedeutung von PTHrP und Bone Sialoprotein für die Entstehung von Knochenmetastasen beim Mammakarzinom [PTHrP and bone sialoprotein as prognostic markers for developing bone metastases in breast cancer patients]. Zentralbl Gynakol 2006;128(6):330-5.

53. Chaplet M, Waltregny D, Detry C, Fisher LW, Castronovo V, Bellahcène A. Expression of dentin sialophosphoprotein in human prostate cancer and its correlation with tumor aggressiveness. Int J Cancer 2006;118(4):850-6.

54. Costa LA, Alho I, Casimiro S, Oliveira AG, Luís I, Fernandes A, et al. Markers of bone turnover (ICTP, NTX) and serum matrix metalloproteinase 1 (MMP1) as prognostic markers in breast cancer patients (BC) with bone metastases (BM) treated with bisphosphonates (BP). J Clin Oncol 2008;26(15_Suppl):47s, [abstract 1024].

55. Smith MR, Kabbinavar F, Saad F, Hussain A, Gittelman MC, Bilhartz DL, et al. Natural history of rising serum prostate-specific antigen in men with castrate non-metastatic prostate cancer. J Clin Oncol 2005;23(13):2918-25.

56. Naruse K, Yamada Y, Aoki S, Taki T, Nakamura K, Tobiume $\mathrm{M}$, et al. Lactate dehydrogenase is a prognostic indicator for prostate cancer patients with bone metastasis. Hinyokika Kiyo 2007;53(5):287-92.

57. Kikuchi E, Nakashima J, Ishibashi M, Ohigashi T, Oya M, Nakagawa K, et al. Usefulness of alpha1-antichymotrypsin-PSA complex for predicting bone metastases of prostate cancer. Urology 2006;68(2):371-5.

58. Nishio Y, Yamada Y, Kokubo H, Nakamura K, Aoki S, Taki T, et al. Prognostic significance of immunohistochemical expression of the HER-2/neu oncoprotein in bone metastatic prostate cancer. Urology 2006;68(1):110-5. 